\title{
SPECTRAL DYNAMIC ANALYSIS OF A STATIONARY JACK-UP PLATFORM
}

\author{
Bogdan Rozmarynowski \\ Gdańsk University of Technology, Poland
}

\begin{abstract}
The paper refers to the dynamic short-term response analysis of the Baltic steel drilling platform (see Fig.2) in a random sea-state represented by one-dimensional wave spectrum proposed by Striekalov and Massel, which is recommended for the Baltic Sea area. The Baltic drilling platform is a jack-up type platform for the exploration and exploitation of oil under the Baltic Sea. The presented analysis deals with the stationary phase of the platform life when its legs are fixed in the sea bottom. The submerged elements of jack-up platforms are relatively slender, thus to assess the in-line wave forces a modified Morison equation is justified. The application of frequency transfer functions to offshore vibration systems leads to structural response spectra whose input is defined by the wave elevation and wind velocity spectra. The analysis can be applied also to support structures for offshore wind turbines.
\end{abstract}

Keywords: offshore structures, random variables, structural reliability, simulation methods

\section{INTRODUCTION}

Offshore platforms are structural engineering systems designed and erected to withstand severe sea conditions including dynamic wave and wind loads. The paper follows the research of [4] and deals with dynamic analysis of a Baltic movable jack-up drilling platform. Stationary work phase of a jack-up platform assumes its fixed supporting in the seabed. The soil subsystem interacts both ways with the structure through the foundation, therefore a soilstructure interaction problem is analysed here. The analytical method is aimed at computing frequency transfer functions linked with the input wave elevation and wind fluctuation velocity spectra (see Fig. 7 and Fig. 8) in order to obtain structural response spectra. This concept of analyzing the offshore structural engineering systems assumes linearized relations between structural input and output. A general computational procedure aimed at response spectra (nodal displacements, cross-sectional forces, stresses) is presented in Fig. 6, Appendix 1.

The model of a jack-up platform assumes its support by submerged cylindrical members (see Fig. 2), too slender to significantly alter the incident wave field, thus application of the modified Morison equation is justified. The equation defines the wave force normal to the cylinder axis, collinear with the wave propagation direction. In a probabilistic approach the Morison function is affected by Gaussian wave particle kinematics [1].

In the case of slender cylindrical members subjected to sea waves action the wave-structure interaction problem is inherently nonlinear as shown in Fig. 1. The following relations hold: $H / D>1, D / L>0.2$ where $H$ is the wave height, $L$ is the wave length, $D$ is the cylinder diameter (potential theory is applied, no diffractions effects).

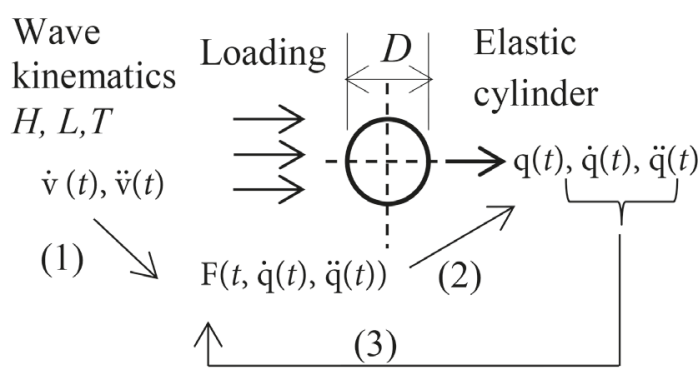

Fig. 1. Scheme of wave-structure interaction problem 
The modified Morison equation (this version includes the relative wave kinematics due to moving cylinder), cf. [2], [3], [4]) reads:

$$
\begin{aligned}
& d \mathbf{F}(s, t)=d \mathbf{F}_{p}(s, t)+d \mathbf{F}_{a}(s, t)+d \mathbf{F}_{d}(s, t)= \\
& =\rho \frac{\pi D^{2}}{4} C_{M}^{\prime} \frac{\partial \mathbf{v}_{n}(s, t)}{\partial t} d s+ \\
& +\rho \frac{\pi D^{2}}{4} C_{M}^{\prime \prime}\left(\frac{\partial \mathbf{v}_{n}(s, t)}{\partial t}-\frac{\partial \dot{\mathbf{q}}_{n}(s, t)}{\partial t}\right) d s+ \\
& +\frac{1}{2} \rho D C_{D}\left[\left(\mathbf{v}_{n}(s, t)-\dot{\mathbf{q}}_{n}(s, t)\right)\left|\mathbf{v}_{n}(s, t)-\dot{\mathbf{q}}_{n}(s, t)\right|\right] d s
\end{aligned}
$$

Here $d \mathbf{F}(s, t)$ is the differential wave force normal to the axis of the cylinder of the diameter $D$, co-linear with the direction of wave propagation on a length $d s, s$ denotes the variable along the element length, $\rho$ is the density of water, $\mathbf{v}_{n}(s, t)$ is the water particle velocity in the undisturbed wave process, subscript " $n$ " denotes normal to the cylinder axis, $C_{M}^{\prime}, C_{M}^{\prime \prime}$, $C_{D}$ are empirical coefficients affected by the cross-sectional shapes, Reynolds and Keulegan-Carpenter Numbers and relative roughness ([5], 12]), $\mathbf{q}(s, t)$ is the cylinder response (displacement), $\partial(\bullet) / \partial t$ denotes the partial time derivative.

\section{ASSUMPTIONS AND SOLUTION TECHNIQUES}

The following assumptions with regard to two cases of the stochastic approach to the problem presented in Fig. 1, are considered:

\section{Linear stochastic problem:}

- deterministic soil-structure interaction which is represented by a system of springs and dash-pots connecting the legs of the structure and subsoil. The number of these connecting elements depends on the subsoil penetration depth of the structure leg ends,

- linearization of the velocity term in Eq. (1) is carried out by means of the least-square technique or stochastic averaging method (cf. [8], [10]),

- wave and wind loads are considered stationary Gaussian zero-mean ergodic processes, mutually independent,

- the equations of motion of the problem are solved in a reduced modal space.

II. Non-linear stochastic problem:

- non-deterministic soil-structure interaction problem is considered where the soil shear modulus $G$ is a Gaussian random variable,

- the spring stiffnesses are linear functions of $G$, whereas the dash-pots are non-linear functions, it eventually yields non-Gaussian random variables [6],

- no vortex- induced oscillations are included in the analysis.

In the presented paper the following solution techniques are applied:

1. Modal transformation of the equation of motion:

$$
\mathbf{M} \ddot{\mathbf{q}}(t)+\mathbf{C} \dot{\mathbf{q}}(t)+\mathbf{K q}(t)=\mathbf{P}(t, \dot{\mathbf{q}}(t), \ddot{\mathbf{q}}(t))
$$

where $\mathbf{M}, \mathbf{C}, \mathbf{K}$ are the overall mass, damping and stiffness matrices $n \times n$, respectively, $\mathbf{q}(t)$ and $\mathbf{P}(t, \dot{\mathbf{q}}(t), \ddot{\mathbf{q}}(t))$ are the global displacement and applied force vectors $n \times 1$, $\mathbf{M}=\mathbf{M}_{K}+\mathbf{M}_{S}+\mathbf{M}_{H}, \mathbf{C}=\mathbf{C}_{K}+\mathbf{C}_{S}+\mathbf{C}_{H}^{R}, \mathbf{K}=\mathbf{K}_{K}+\mathbf{K}_{S}$, where $\mathbf{M}_{K}, \mathbf{C}_{K}, \mathbf{K}_{K}$ are the structural parts of matrices $\mathbf{M}_{S}, \mathbf{C}_{S}, \mathbf{K}_{S}$, show the soil impact $\mathbf{M}_{H}, \mathbf{C}_{H}^{R}$ denote hydrodynamic parts of matrices, an upper $\operatorname{dot} \mathbf{P}(t, \dot{\mathbf{q}}(t), \ddot{\mathbf{q}}(t))$ denotes a time derivative, is the force vector due to hydrodynamic and wind loads:

$$
\mathbf{P}(t, \dot{\mathbf{q}}(t), \ddot{\mathbf{q}}(t))=\mathbf{F}(t, \dot{\mathbf{q}}(t), \ddot{\mathbf{q}}(t))+\mathbf{W}(t, \dot{\mathbf{q}}(t))
$$

where: $\mathbf{F}$ and $\mathbf{W}$ are referred to wave and wind force vectors, respectively.

The eigenvalue problem is formulated by $\mathbf{K} \boldsymbol{\Phi}_{i}=\lambda_{i} \mathbf{M} \boldsymbol{\Phi}_{i}$, where $\lambda_{i}=\omega_{0 i}^{2}$ is the $i$-th eigenvalue (squared natural frequency), $\boldsymbol{\Phi}_{i}$ is the $i$-th eigenvector, $\left(\omega_{01}, \Phi_{1}\right),\left(\omega_{02}, \Phi_{2}\right), \ldots,\left(\omega_{0 n}, \Phi_{n}\right)$ is the eigensolution with the orthogonality relation $\boldsymbol{\Phi}_{i}^{T} \mathbf{M} \boldsymbol{\Phi}_{j}=\left[\delta_{i j}\right]=\mathbf{I}$, where $\delta_{i j}$ is the Kronecker delta, $i, j=1,2 \ldots, n$, is the $n \times n$ unit matrii.

Let $\boldsymbol{\Phi}_{h}=\left[\boldsymbol{\Phi}_{1}, \boldsymbol{\Phi}_{2}, \ldots \boldsymbol{\Phi}_{h}\right], h \leq n$ constitutes the reduction of the modal space and $\boldsymbol{\Phi}^{T}{ }_{h} \mathbf{M} \boldsymbol{\Phi}_{h}=\mathbf{I}_{h}, \boldsymbol{\Phi}^{T}{ }_{h} \mathbf{K} \boldsymbol{\Phi}_{h}=\boldsymbol{\Omega}^{2}{ }_{h}=\operatorname{diag}\left\{\omega_{01}^{2}, \omega_{02}^{2} \ldots\right.$ $\left.\omega^{2}{ }_{0 h}\right\}$ where $\mathbf{I}_{h}$ is the $h \times h$ unit matrix.

2. Stochastic linearization method [7]:

The nonlinear (velocity) part of the Morison Eq. (1) is:

$$
\mathbf{X}(\dot{\mathbf{x}}|\dot{\mathbf{x}}|)=\left(\mathbf{v}_{n}(s, t)-\dot{\mathbf{q}}_{n}(s, t)\right)\left|\mathbf{v}_{n}(s, t)-\dot{\mathbf{q}}_{n}(s, t)\right|
$$

This term may be linearized with a tendency to minimize the mean-square error:

$\mathbf{X}(\dot{\mathbf{x}}|\dot{\mathbf{x}}|) \rightarrow \mathbf{A} \dot{\mathbf{x}}$, where $\mathbf{e}=\mathbf{X}(\dot{\mathbf{x}}|\dot{\mathbf{x}}|) \mathbf{A} \dot{\mathbf{x}}-$ and

$$
e_{i}^{2}=E\left[\left(\dot{x}_{i}\left|\dot{x}_{i}\right|-A_{i i} \dot{x}_{i}\right)^{2}\right] \rightarrow \min
$$

The condition (5) gives the result:

$$
\frac{\partial e_{i}^{2}}{\partial A_{i i}}=0 \Rightarrow A_{i i}=\frac{E\left(\dot{x}_{i}^{2}\left|\dot{x}_{i}\right|\right)}{E\left(\dot{x}_{i}^{2}\right)}=\sqrt{\frac{8}{\pi}} \sigma_{\dot{x}_{i}}
$$

The last term of the Morison equation (1) denotes the drag force. In view of the approximation (6) one gets the force equivalent to the drag force ([6], [8]), given by:

$$
d \widetilde{\mathbf{F}}_{d}(s, t)=\frac{1}{2} \rho D C_{D} \sqrt{\frac{8}{\pi}} \cdot \sigma_{\mathbf{v}_{n}-\dot{\mathbf{q}}_{n}}\left(\mathbf{v}_{n}(s, t)-\dot{\mathbf{q}}_{n}(s, t)\right) d s
$$

where: $\sigma_{\mathbf{v}_{n}-\dot{\mathbf{q}}_{n}}$ is a standard deviation of the relative velocity (assumed that $\sigma_{\mathbf{v}_{n}-\dot{\mathbf{q}}_{n}} \approx \sigma_{\mathbf{v}_{n}}$ if the condition $\left|\mathbf{v}_{n}(s, t)\right|>>\left|q_{n}(s, t)\right|$ is satisfied).

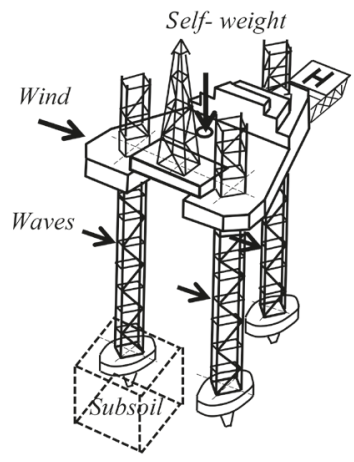

Fig. 2. General view of the platform 
3. The perturbation method (cf [6], [9]):

Let the total mass, damping and stiffness matrices in Eq. (2) take the form:

$$
\begin{aligned}
\mathbf{M}=\mathbf{M}_{0}+\varepsilon \mathbf{M}_{1}(\gamma), \mathbf{C}=\mathbf{C}_{0}+\varepsilon \mathbf{C}_{1}(\gamma), \mathbf{K}=\mathbf{K}_{0}+\varepsilon \mathbf{K}_{1}(\gamma), \\
\mathbf{q}(\gamma, t)=\mathbf{q}_{0}(\gamma, t)+\varepsilon \mathbf{q}_{1}(\gamma, t)
\end{aligned}
$$

where: $\varepsilon$ is the small parameter $|\varepsilon|<<1, \gamma$ is an elementary event.

It yields the following set of equations:

$$
\begin{gathered}
\mathbf{M}_{0} \ddot{\mathbf{q}}_{0}(\gamma, t)+\mathbf{C}_{0} \dot{\mathbf{q}}_{0}(\gamma, t)+\mathbf{K}_{0} \mathbf{q}_{0}(\gamma, t)=\mathbf{P}(\gamma, t) \\
\mathbf{M}_{0} \ddot{\mathbf{q}}_{1}(\gamma, t)+\mathbf{C}_{0} \dot{\mathbf{q}}_{1}(\gamma, t)+\mathbf{K}_{0} \mathbf{q}_{1}(\gamma, t)= \\
-\left[\mathbf{M}_{1}(\gamma) \ddot{\mathbf{q}}_{0}(\gamma, t)+\mathbf{C}_{1}(\gamma) \dot{\mathbf{q}}_{0}(\gamma, t)+\mathbf{K}_{1}(\gamma) \mathbf{q}_{0}(\gamma, t)\right]= \\
\mathbf{Z}(\gamma, t)
\end{gathered}
$$

where $\mathbf{M}_{1}(\gamma)=\operatorname{diag}\left[M_{\text {Sii }}(\gamma)\right], \mathbf{C}_{1}(\gamma)=\operatorname{diag}\left[C_{S_{i i}}(\gamma)\right]$, $\mathbf{K}_{1}(\gamma)=\operatorname{diag}\left[K_{S i i}(\gamma)\right]$ define diagonal elements of the mass, damping and stiffness matrices due to soil, $\mathbf{Z}(\gamma, t)$ is the vector of stochastic stationary process in the wide sense, ergodic in the mean but not in the correlation. The above given expressions are linear stochastic equations with deterministic parameters and stochastic excitations.

4. The stochastic averaging method:

A diffusion Markov vector process with stationary solution of the Itộ stochastic differential equations with respect to the Wiener process (with orthogonal increments) is applied - cf. [8], [10]).

By transforming Eq. (2) into reduced modal space, the $i$-th equation of motion may be written as follows:

$$
\ddot{y}_{i}(t)+\omega_{0 i}^{2} y_{i}(t)=\varepsilon N_{i}\left[\dot{y}_{i}(t), v_{n}(t), t\right]
$$

where: $N_{i}$ is the $i$-th element of the vector $\mathbf{N}_{h}=\mathbf{R}_{h}-\boldsymbol{\beta}_{h}^{R} \dot{\mathbf{y}}_{h}-\boldsymbol{\beta}_{h}^{F} \dot{\mathbf{y}}_{h}$, $i=1,2, \ldots, h \leq n, \varepsilon$ is the small parameter, $\mathbf{R}_{h}=\boldsymbol{\Phi}_{h}^{T}\left[\mathbf{K}_{M} \dot{\mathbf{v}}_{n}+\right.$ $\left.\mathbf{K}_{D} \mathbf{T}_{1}\left(v_{n}\right)\right], \boldsymbol{\beta}_{h}^{R}=\boldsymbol{\Phi}_{h}^{T}\left(\mathbf{C}_{K}+\mathbf{C}_{H}^{R}\right) \boldsymbol{\Phi}_{h}, \boldsymbol{\beta}_{h}^{F}=\boldsymbol{\Phi}_{h}^{T}\left[2 \mathbf{K}_{D} \mathbf{T}_{2}\left(v_{n}\right)\right] \boldsymbol{\Phi}_{h}$

$$
\begin{aligned}
& \mathbf{T}_{1}\left(v_{n}\right)=\operatorname{diag}\left\{\left|v_{n j}\right| v_{n j}\right\}= \\
& =\operatorname{diag}\left\{\sqrt{2 / \pi} \sigma_{v_{n j}} v_{n j}+1 / 3 \sigma_{v_{n j}}^{-1} \sqrt{2 / \pi} v_{n j}^{3}\right\}, \\
& \mathbf{T}_{2}\left(v_{n}\right)=\operatorname{diag}\left\{1 / 2 \sigma_{v_{n j}}^{-1} \sqrt{2 / \pi}\left(v_{n j}^{2}-\sigma_{v_{n j}}^{2}\right)\right\}, \\
& \mathbf{C}_{H}^{R}=\operatorname{diag}\left\{\sqrt{8 / \pi} K_{D j} \sigma_{v_{n j}}\right\}, j=1,2 \ldots, n, \\
& y_{i} \in \mathbf{y}_{h}=\boldsymbol{\Phi}_{h}^{T} \mathbf{q},
\end{aligned}
$$

$\mathbf{K}_{M}=\rho C_{M} \mathbf{V}, C_{M}=C_{M}^{\prime}+C^{\prime \prime}{ }_{M}, \mathbf{K}_{D}=1 / 2 C_{D} \mathbf{A}, \mathbf{V}$ and $\mathbf{A}$ denote diagonal matrices of the volume and area of the submerged elements normal to the wave particle velocity.

Applying the Krylov - Bogolubov transformation of the joint response process $\left(y_{i}, \dot{y}_{i}\right)$ to a pair of slowly varying processesone $\left(A_{i}, \phi_{i}\right)$ gets:

$$
\begin{aligned}
& y_{i}(t)=A_{i}(t) \cos \Theta_{i}(t), \\
& \dot{y}_{i}(t)=A_{i}(t) \omega \sin \Theta_{i}(t)
\end{aligned}
$$

where: $A_{i}(t)$ is the amplitude envelope process and $\Theta_{i}(t)=$ $\omega_{0 i} t+\varphi_{i}(t), \varphi_{i}(t)$ indicates the phase process, provided that the condition $\dot{A}_{i}(t)=\dot{\varphi}_{i}(t) A_{i}(t) \sin \Theta_{i}(t) / \cos \Theta_{i}(t)$ is satisfied.

By substituting Eqs (11) into (10), the standard equations are:

$$
\begin{aligned}
& \dot{A}_{i}(t)=\varepsilon G_{i}\left[A, \varphi, v_{n}(t), t\right] \\
& \dot{\varphi}_{i}(t)=\varepsilon Z_{i}\left[A, \varphi, v_{n}(t), t\right]
\end{aligned}
$$

where:

$$
\begin{aligned}
& G_{i}\left[A, \varphi, v_{n}(t), t\right]= \\
& =-\left(\frac{1}{\omega_{0 i}}\right) N_{i}\left[-\omega_{0 i} A_{i} \sin \Theta_{i}, v(t), t\right] \sin \Theta_{i}= \\
& =G_{R i}[A, \varphi, t]+G_{F i}\left[A, \varphi, v_{n}(t), t\right] \\
& Z_{i}\left[A, \varphi, v_{n}(t), t\right]= \\
& =-\left(\frac{1}{\omega_{0 i} A_{i}}\right) N_{i}\left[-\omega_{0 i} A_{i} \sin \Theta_{i}, v(t), t\right] \cos \Theta_{i}= \\
& =Z_{R i}[A, \varphi, t]+Z_{F i}\left[A, \varphi, v_{n}(t), t\right]
\end{aligned}
$$

In the above given expressions $G_{R i}$ and $Z_{R i}, G_{F i}$ and $Z_{F i}$ represent the regular and fluctuation parts of the corresponding functions, respectively.

The amplitudes $A_{i}$ and phases $\varphi_{i}$ are considered elements of a multi-dimensional function $\mathbf{X}(t)=\left[A_{i}(t), \varphi_{i}(t), \ldots, A_{h}(t), \varphi_{h}(t)\right]^{T}$.

It can be proved that the response of the Eq. (10) tends to a multi-dimensional diffusive Markov process if the conditions of the Khasminski limit theorem ([20]) are satisfied. The Itộ stochastic differential equation is one of the methods for the analysis of Markov processes. In the case of a vector process $\mathbf{X}(t)$ it holds:

$$
d \mathbf{X}(t)=\mathbf{m}[\mathbf{X}(t)] d t+\boldsymbol{\sigma}[\mathbf{X}(t)] d \mathbf{W}(t)
$$

where $\mathbf{m}[\mathbf{X}(t)]$ is a drift coefficient, $\boldsymbol{\sigma}[\mathbf{X}(t)]$ a diffusion coefficient matrix, $\mathbf{W}(t)$ denotes the Wiener process (representing an independent Brownian motion process).

It can be proved that the equations for amplitudes are uncoupled from ones referring to phases (cf. [8], [19]). The set of Itộ equations (13) takes the form:

$$
\begin{aligned}
& d A_{i}(t)=m_{i}^{A}(A) d t+\sum_{j=1}^{h} \sigma_{i j}^{A} d W_{j}^{A}(t) \\
& d \varphi_{i}(t)=m_{i}^{\varphi}(\varphi) d t+\sum_{j=1}^{h} \sigma_{i j}^{\varphi} d W_{j}^{\varphi}(t)
\end{aligned}
$$

where $W_{j}^{A}, W_{j}^{\varphi}$ are independent Wiener processes, $m_{i}^{A}, m_{i}^{\varphi}$ and $\sigma_{i j}^{A}, \sigma_{i j}^{\varphi}$ are drift and diffusion coefficients, respectively, $i=1,2, \ldots, h$.

The details related to the Khasminski limit theorem are fully developed in [8]. 


\section{STRUCTURAL TRANSFER FUNCTION}

This function can be derived directly from the dynamic equilibrium Eq.(2).

The force vector depends on structural kinematics, in this form the vector generates the nonlinear damping term in Eq. (1). For the linear case the equation can be written in frequency domain:

$$
\begin{aligned}
& \frac{1}{2 \pi} \int_{-\infty}^{\infty}\left(\mathbf{K}-\omega^{2} \mathbf{M}+i \omega \mathbf{C}\right) e^{i \omega t} d \overline{\mathbf{Q}}(\omega)= \\
& =\frac{1}{2 \pi} \int_{-\infty}^{\infty} e^{i \omega t} d \overline{\mathbf{P}}(\omega)
\end{aligned}
$$

where the following transformations are defined:

$$
\begin{aligned}
& \mathbf{q}(t)=\frac{1}{2 \pi} \int_{-\infty}^{\infty} e^{i \omega t} d \overline{\mathbf{Q}}(\omega), \\
& \mathbf{P}(t)=\frac{1}{2 \pi} \int_{-\infty}^{\infty} e^{i \omega t} d \overline{\mathbf{P}}(\omega), i=\sqrt{-1}
\end{aligned}
$$

The equation (16) can be stated in the form:

$$
d \overline{\mathbf{Q}}(\omega)=\mathbf{H}_{Q P}(\omega) d \overline{\mathbf{P}}(\omega)
$$

in which $\mathbf{H}_{O P}(\omega)=\left(\mathbf{K}-\omega^{2} \mathbf{M}+i \omega \mathbf{C}\right)^{-1}$ is the structural transfer function matrix $(n \times n)$.

In the reduced modal space $Q \rightarrow Y$, the function $\mathbf{H}_{Q P}(\omega)$ takes an approximate form:

$$
\begin{aligned}
& \hat{\mathbf{H}}_{Y P}(\omega)=\left[\boldsymbol{\Phi}_{h}^{T}\left(\mathbf{K}-\omega^{2} \mathbf{M}+i \omega \mathbf{C}\right) \boldsymbol{\Phi}_{h}\right]^{-1}= \\
& =\sum_{r=1}^{h} H_{r}(\omega) \boldsymbol{\Phi}_{h r} \boldsymbol{\Phi}_{h r}^{T} \\
& H_{r}(\omega)=\frac{1}{k_{r}} \frac{\omega_{0 r}^{2}}{\left(\omega_{0 r}^{2}-\omega^{2}\right)+2 i \xi_{r} \omega_{0 r} \omega},
\end{aligned}
$$

where:

$k_{r}$ is the generalized stiffness, $\omega_{0 r}$, $\xi_{r}$ are the $r$-th natural frequency and the damping ratio, respectively, $h \leq n$ denotes the number of modes $\Phi_{h}$ in the reduced space.

\section{WAVE TRANSFER FUNCTION}

In the case o fsufficiently slender elements loaded by sea waves the Morison equation is applicable, it can be written in vector notation:

$$
\mathbf{F}(t)=f(\beta)\left[\mathbf{K}_{M} \dot{\mathbf{v}}_{n}(t)+\mathbf{K}_{D} \mathbf{v}_{n}(t)\right]
$$

where $\dot{\mathbf{v}}_{n}=\mathbf{a}_{n}$, is the acceleration vector,

$$
\mathbf{K}_{D}=1 / 2 \bar{C}_{D} \mathbf{A}, \bar{C}_{D}=C_{D} \sqrt{\frac{8}{\pi}} \sigma_{\mathbf{v}_{n}},
$$

$\sigma_{\mathbf{v}_{n}}$ is a standard deviation of horizontal velocity of water in the wave direction, $f(\beta)$ is a function of wave direction flow angle.

The force (20) can be stated in the frequency domain:

$$
\mathbf{F}(\omega)=\mathbf{H}_{F \eta}(\omega) \eta(\omega)
$$

where: $\eta(\omega)$ denotes water elevation considered the input function, $\mathbf{H}_{F \eta}(\omega)$ is the transfer function of the wave force (cf. [11]) according to the Morison equation:

$$
\mathbf{H}_{F \eta}(\omega)=f(\beta)\left[\mathbf{K}_{M}+i \omega \mathbf{K}_{D}\right] \mathbf{H}_{v \eta}(\omega)
$$

in which $\mathbf{H}_{v \eta}(\omega)$ is the transfer function vector between the normal water velocity and the water elevation.

The transfer function vector of the wave force given by Eq. (22) is defined in global coordinates for an entire structure after an assembly process of transfer functions of member forces. Given a particular element " $e$ " whose length is $l$ the wave force shows exponential distribution in vertical direction and harmonic in horizontal direction. The transfer functions of equivalent wave forces at member ends are calculated by assuming cubic polynomial functions to approximate member deformations ([12]). These transfer functions are given the following vector notation with respect to a member " $e$ ":

$$
\mathbf{H}_{F \eta \mid e}^{L}(\omega)_{e}=\int_{0}^{l_{e}} \mathbf{N}_{e}^{T} \mathbf{H}_{F \eta}^{e}(\omega) d x
$$

where: $\mathbf{N}_{e}$ describes shape function matrix of member deformations, $l_{e}$ is the member length, $x$ denotes a variable along the member axis, the superscript $L$ indicates local coordinates. While an assembled system is considered this vector is transformed to global coordinates according to standard finite elements method (FEM) procedures.

\section{RESPONSE SPECTRA}

The response displacement spectral density function can be presented in the form:

$$
\mathbf{S}_{y y}(\omega)=\hat{\mathbf{H}}_{Y P}(\omega) \hat{\mathbf{S}}_{P P}(\omega)\left(\hat{\mathbf{H}}_{Y P}^{*}(\omega)\right)^{T}
$$

where: $\hat{\mathbf{H}}_{Y P}$ is given by Eq. (19),

$$
\hat{\mathbf{S}}_{P P}(\omega)=\boldsymbol{\Phi}_{h}^{T} \mathbf{S}_{P P}(\omega) \boldsymbol{\Phi}_{h}, \mathbf{S}_{P P}(\omega)=\mathbf{S}_{F F}(\omega)+\mathbf{S}_{W W}(\omega) .
$$

Here $\mathbf{S}_{F F}(\omega)$ and $\mathbf{S}_{W W}(\omega)$ correspond to waves and wind spectra, respectively.

The cross-spectral density of the wave load is:

$$
\begin{aligned}
& \mathbf{S}_{F_{m} F_{n}}(\omega)=\mathbf{H}_{F \eta \mid m}^{T}(\omega) \mathbf{H}_{F \eta \mid n}(\omega)= \\
& =\frac{\rho^{2} \pi^{2} D_{m}^{2} D_{n}^{2} C_{M m} C_{M n}}{16} \mathbf{S}_{v_{m} v_{v}}(\omega)+ \\
& +\frac{\rho^{2} D_{m} D_{n} \bar{C}_{D m} \bar{C}_{D n}}{4} \mathbf{S}_{v_{m} v_{n}}(\omega)+ \\
& +\frac{\rho^{2} \pi}{8}\left(C_{M m} \bar{C}_{D n} D_{m}^{2} D_{n}-C_{M n} \bar{C}_{D m}\right) \mathbf{S}_{v_{m} v_{n}}(\omega)
\end{aligned}
$$

in which " $m$ " and " $n$ " are points of the structure with their global coordinates $\left\{x_{m}, y_{m}, z_{m}\right\}$ and $\left\{x_{n}, y_{n}, z_{n}\right\}$, respectively, $D_{m}$ and $D_{n}$ denote diameters of structural submerged members at these points, 


$$
\begin{gathered}
\mathbf{S}_{v_{m} v_{n}}(\omega)=\mathbf{H}_{v \eta}^{T}(\omega) \mathbf{H}_{v \eta}(\omega)= \\
=\omega^{2} S_{\eta \eta}(\omega) \mathbf{D}\left(k, z_{m}, z_{n}\right) \cdot \\
\cdot \exp [-i k(\Delta x \cos \beta+\Delta y \sin \beta)] \\
\mathbf{D}\left(k, z_{m}, z_{n}\right)=\mathbf{A}\left(k, z_{m}\right) \mathbf{A}^{T}\left(k, z_{n}\right) \\
\mathbf{A}(k, z)=\frac{1}{\sinh (k d)}\left\{\begin{array}{c}
\cos \beta \cosh (k z) \\
\sin \beta \cosh (k z) \\
i \sinh (k z)
\end{array}\right\} \\
\mathbf{S}_{\dot{v}_{m} \dot{v}_{n}}(\omega)=\omega^{2} \mathbf{S}_{v_{m} v_{n}}(\omega) \\
\mathbf{S}_{\dot{v}_{m} v_{n}}(\omega)=i \omega \mathbf{S}_{v_{m} v_{n}}(\omega)
\end{gathered}
$$

where: $S_{\eta \eta}(\omega)$ in Eq. (26) denotes water elevation spectrum (e.g. the Massel-Striekalov spectrum [13], see Appendix 2, Fig. 4), $k$ stands for the wave number, $k=2 \pi / L_{w}$, where $L_{w}$ is the wave length, $d$ is the water depth, $\beta$ is long-crested - wave direction flow angle, $\Delta x=x_{m}-x_{n}, \Delta y=y_{m}-y_{n}$.

The cross-spectral density of the wind load can be written as follows:

$$
\begin{aligned}
& S_{W m W n}(\omega)=\rho_{A}^{2} C_{D A m} C_{D A n} A_{m} A_{n} \cdot \\
& \cdot\left[\left|\bar{v}_{m}\right|\left|\bar{v}_{n}\right|+\frac{1}{2 \pi} \sigma_{v_{m}^{\prime} v_{m}^{\prime}} \sigma_{v_{n}^{\prime} v_{n}^{\prime}}+\sqrt{\frac{2}{\pi}}\left|\bar{v}_{m}\right| \sigma_{v_{n}^{\prime} v_{n}^{\prime}}\right] S_{v_{m}^{\prime} v_{n}^{\prime}}
\end{aligned}
$$

where: $\rho_{A}$ is the air density, $C_{D A m}, C_{D A n}, A_{m}, A_{n},\left|\bar{v}_{m}\right|,\left|\bar{v}_{n}\right|, \sigma \sigma_{v^{\prime}}{ }^{v^{\prime}}{ }_{n}$ and $\sigma v^{\prime}{ }_{m} v_{m}^{\prime}$ are the wind drag coefficients, windward areas, mean velocities, standard deviations at the points " $m$ " and " $n$ "lying in the vertical plane perpendicular to the mean-wind velocity, respectively, $S_{v^{\prime}}{ }_{m}{ }^{\prime}{ }_{n}$ is the cross-spectral density of the along-wind fluctuating velocity for the mentioned points:

$$
S_{v_{m}^{\prime} v_{n}^{\prime}}(\omega)=S_{v_{m}^{\prime} v_{n}^{\prime}}^{R E}(\omega)+i S_{v_{m}^{\prime} v_{n}^{\prime}}^{I M}(\omega)
$$

where :

$$
\begin{aligned}
& S_{v_{m}^{\prime} v_{n}^{\prime}}^{R E}(\omega)=\left|S_{v_{m}^{\prime} v_{n}^{\prime}}^{C}(\omega)\right| \sqrt{S_{v_{m}^{\prime} v_{m}^{\prime}}(\omega) S_{v_{n}^{\prime} v_{n}^{\prime}}(\omega)} \cos \theta_{v_{m}^{\prime} v_{n}^{\prime}}(\omega) \\
& S_{v_{m}^{\prime} v_{n}^{\prime}}^{I M}(\omega)=\left|S_{v_{m}^{\prime} v_{n}^{\prime}}^{C}(\omega)\right| \sqrt{S_{v_{m}^{\prime} v_{m}^{\prime}}(\omega) S_{v_{n}^{\prime} v_{n}^{\prime}}(\omega)} \sin \theta_{v_{m}^{\prime} v_{n}^{\prime}}(\omega) \\
& \theta_{v_{m}^{\prime} v_{n}^{\prime}}(\omega)=\operatorname{arctg}\left[S_{v_{m}^{\prime} v_{n}^{\prime}}^{I M}(\omega) / S_{v_{m}^{\prime} v_{n}^{\prime}}^{R E}(\omega)\right]=c \omega r_{m n} / \bar{v} \\
& S_{v_{m}^{\prime} v_{n}^{\prime}}^{C}(\omega)=\exp \left[-\frac{a b \omega r_{m n} / \bar{v}}{\left(b^{2} \cos ^{2} \beta_{m n}+a^{2} \sin ^{2} \beta_{m n}\right)}\right]
\end{aligned}
$$

$\bar{v}$ is the mean speed at the reference height, $\beta_{m n}$ is the angle between the direction $(m, n)$ and the horizontal, $r_{m n}$ is the distance between the points $m$ and $n, a, b, c$ are empirical coefficients, $S_{v_{m}^{\prime} v_{m}^{\prime}}$ and $S_{v_{n}^{\prime} v_{n}^{\prime}}$ are spectral density functions (cf. (A2), Appendix 3) at two points $m$ and $n$.

The calculation of transfer functions $\hat{\mathbf{H}}_{Y P}$ is a time consuming process and the following relation is applied:

$$
\hat{\mathbf{Z}}_{Y P}(\omega) \hat{\mathbf{H}}_{Y P}(\omega)=\mathbf{I}_{h}
$$

here $\mathbf{I}_{h}$ is the unit matrix of $h \times h$ dimension.

Due to the above mentioned statement the matrices may be presented in the form:

$$
\hat{\mathbf{Z}}_{Y P}(\omega)=\mathbf{A}+i \mathbf{B}, \hat{\mathbf{H}}_{Y P}(\omega)=\mathbf{D}+i \mathbf{E}
$$

where: $\mathbf{A}=\boldsymbol{\Phi}_{h}^{T}\left(\mathbf{K}-\omega^{2} \mathbf{M}\right) \boldsymbol{\Phi}_{h}, \mathbf{B}=\boldsymbol{\Phi}_{h}^{T} \omega \mathbf{C} \boldsymbol{\Phi}_{h}, \mathbf{D}=\operatorname{Re} \hat{\mathbf{H}}_{Y P}(\omega)$, $\mathbf{E}=\operatorname{Im} \hat{\mathbf{H}}_{Y P}(\omega)$

The set of equations (31), (32) in matrix form reads:

$$
\left[\begin{array}{cc}
\mathbf{A} & -\mathbf{B} \\
-\mathbf{B} & -\mathbf{A}
\end{array}\right]\left[\begin{array}{l}
\mathbf{D} \\
\mathbf{E}
\end{array}\right]=\left[\begin{array}{c}
\mathbf{I}_{h} \\
\mathbf{0}
\end{array}\right]
$$

where the right-hand side of (34) is composed of $2 h \times 2 h$ vectors and the $m$-th vector can be stated as follows:

$$
\left[\mathbf{I}_{h}^{m}, \mathbf{0}\right]^{T}=\left[\left(0,0, \ldots 1_{m}, 0, \ldots\right): \mathbf{0}\right]_{1 \times 2 h}
$$

whileis $1_{m}$ unity located at the $m$-th place.

By introducing the following notations: $k_{i i}=\boldsymbol{\Phi}_{h}^{T} \mathbf{K} \Phi_{h}$ and $m_{i i}=\boldsymbol{\Phi}_{h}^{T} \mathbf{M} \boldsymbol{\Phi}_{h}, c_{i i}=\boldsymbol{\Phi}_{h}^{T} \mathbf{C} \boldsymbol{\Phi}_{h}$ the solution of Eq. (32) can be stated as follows:

$$
\begin{aligned}
& \operatorname{Re} H_{m i}=\frac{k_{i i}-\omega^{2} m_{i i}}{\left(k_{i i}-\omega^{2} m_{i i}\right)^{2}+c_{i i}^{2} \omega^{2}}=\frac{\omega_{0 i}^{2}-\omega^{2}}{\left(\omega_{0 i}^{2}-\omega^{2}\right)^{2}+4 \xi_{i}^{2} \omega_{0 i}^{2} \omega^{2}} \\
& \operatorname{Im} H_{m i}=\frac{-\omega c_{i i}}{\left(k_{i i}-\omega^{2} m_{i i}\right)^{2}+c_{i i}^{2} \omega^{2}}=\frac{-2 \xi_{i} \omega_{0 i} \omega}{\left(\omega_{0 i}^{2}-\omega^{2}\right)^{2}+4 \xi_{i}^{2} \omega_{0 i}^{2} \omega^{2}}
\end{aligned}
$$

in which $m, i=1,2, \ldots .2 h, \omega$ and $\omega_{0 i}$ denote spectrum frequency and $i$-th structural natural frequency, respectively.

\section{THE SUBSOIL PROPERTIES}

The subsoil parameters are assumed deterministic or random (cf. [4], [8], [16]), resulting in linear or non-linear stochastic problem (elements of stiffness and damping matrices are deterministic or random). In both variants the subsoil is assumed a homogeneous linear half-space defined by shear modulus $G$ and Poisson's ratio $v$. It is assumed that in the second variant of the subsoil model the shear modulus is random, whereas the Poisson's ratio is deterministic. The soil-structure interaction is simulated by a set of springs and dash-pots. The mean values and the variances of the spring stiffnesses are ([cf. 6], [16]):

$$
\begin{aligned}
& \bar{K}_{H}=\frac{8 r_{0}}{2-v} \bar{G}, \bar{K}_{V}=\frac{4 r_{0}}{1-v} \bar{G}, \\
& \bar{K}_{R}=\frac{8 r_{0}^{3}}{3(1-v)} \bar{G}, \bar{K}_{T}=\frac{16 r_{0}^{3}}{3} \bar{G} \\
& \sigma_{K_{H}}^{2}=\frac{64 r_{0}^{2}}{(2-v)^{2}} \sigma_{G}^{2}, \sigma_{K_{V}}^{2}=\frac{16 r_{0}^{2}}{(1-v)^{2}} \sigma_{G}^{2}, \\
& \sigma_{K_{R}}^{2}=\frac{7.11 r_{0}^{6}}{(1-v)^{2}} \sigma_{G}^{2}, \sigma_{K_{T}}^{2}=28.44 r_{0}^{6} \sigma_{G}^{2}
\end{aligned}
$$


where: the superscripts $H, V, R$ and $T$ denote horizontal, vertical, flexural and torsional components of the spring stiffnesses, $r_{0}$ is the radius of the foot circular plate, $\bar{G}$ is the mean value of shear modulus and $\sigma_{G}^{2}$ denotes its variance.

Due to the dash-pot coefficients we denote (cf. [6], [16]):

$$
\begin{aligned}
& \bar{C}_{v}=3.58 r_{0}^{2} \sqrt{\frac{\rho}{1-v}} E\{\sqrt{G}\}, \\
& \bar{C}_{H}=3.05 r_{0}^{2} \sqrt{\frac{\rho}{2-v}} E\{\sqrt{G}\}, \\
& \bar{C}_{M}=0.77 r_{0}^{4} \sqrt{\frac{\rho}{1-v}} E\{\sqrt{G}\}, \\
& \bar{C}_{T}=2.56 r_{0}^{4} \sqrt{\rho E}\{\sqrt{G}\}, \\
& \sigma_{C V}^{2}=\frac{12.82 \rho r_{0}^{4}}{1-v} \bar{G}-\left(\bar{C}_{v}\right)^{2}, \\
& \sigma_{C H}^{2}=\frac{9.33 \rho r_{0}^{4}}{2-v} \bar{G}-\left(\bar{C}_{H}\right)^{2}, \\
& \sigma_{C M}^{2}=\frac{0.59 \rho r_{0}^{8}}{1-v} \bar{G}-\left(\bar{C}_{M}\right)^{2}, \\
& \sigma_{C T}^{2}=6.57 r_{0}^{8} \bar{G}-\left(\bar{C}_{T}\right)^{2} .
\end{aligned}
$$

where : $\quad E\{\sqrt{G}\}=(\bar{G})^{\frac{1}{2}}\left[1-\frac{1}{8} \frac{\sigma_{G}^{2}}{\bar{G}^{2}}-\frac{15}{128} \frac{\left(\sigma_{G}^{2}\right)^{2}}{\bar{G}^{4}}\right]$

is the approximate formula for the mathematical expectation of the shear modulus, assuming its Gaussian distribution.

\section{NUMERICAL EXAMPLE}

A numerical example concerns the existing structure, the Baltic drilling platform. The space beam model of the platform with 12 nodes and 12 beams is shown in Fig. 3 (cf. [4], [6]).

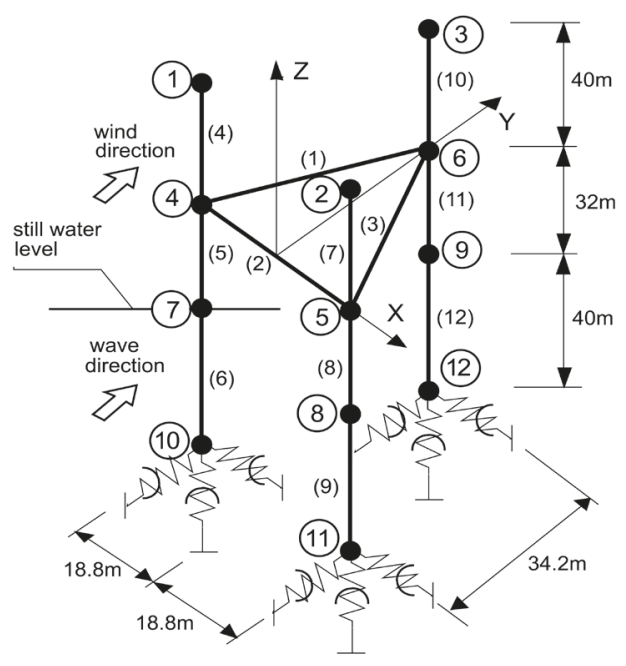

Fig. 3. Discrete model of the platform
Springs and dash-pots in Fig. 5 simulate elastic and damping properties of the subsoil. The platform is subjected to wind and wave loads which are treated as mutually independent stochastic processes.

Structural parameters are collected in Tab. 1 and 2.

Tab. 1. Element stiffnesses

\begin{tabular}{|c|c|c|c|}
\hline Part & $E A[M N]$ & $G_{S}\left[M N m^{2}\right]$ & $E J\left[M N m^{2}\right]$ \\
\hline Deck & $8.40 \cdot 10^{5}$ & $1.94 \cdot 10^{6}$ & $2.53 \cdot 10^{6}$ \\
\hline Legs & $0.79 \cdot 10^{5}$ & $6.86 \cdot 10^{5}$ & $8.93 \cdot 10^{5}$ \\
\hline
\end{tabular}

Tab. 2. Lumped massess

\begin{tabular}{|c|c|}
\hline Node & Mass $[\mathrm{Mg}]$ \\
\hline $1(2,3)$ & 300 \\
\hline $4(5,6)$ & 1800 \\
\hline $7(8,9)$ & 310 \\
\hline $10(11,12)$ & 160 \\
\hline Total & $2570 \times 3=7710$ \\
\hline Hydrodynamic masses & 550 \\
\hline Deck exploitation mass & 1700 \\
\hline
\end{tabular}

Wave, wind and subsoil parameters assumed in the example are as follows:

wave parameters: $\bar{H}=14 m, \bar{T}=10 \mathrm{~s}, \bar{L}=180 \mathrm{~m}, C_{M}^{\prime}=C^{\prime \prime}{ }_{M}$ $=C_{D}=1$, where $\bar{H}, \bar{T}, \bar{L}$ are mean height, mean period and mean length for long-crested waves, respectively, $C_{M}^{\prime}, C^{\prime \prime}{ }_{M}$, $C_{D}$ are empirical inertia and drag coefficients of the Morison equation [15],

wind parameters (see (A2), Appendix 3): $\bar{v}_{10}=40 \mathrm{~m} / \mathrm{s}$, $\mathrm{K}=0.005, a=b=c=1$ where $\bar{v}_{10}$ is the mean wind speed at a height of $10 \mathrm{~m}$ above the surface, $K$ denotes the surface drag coefficient, $a, b, c$ are empirical coefficients in the Davenport formula expressing the cross-spectral density of the alongwind fluctuating velocity [14],

subsoil parameters: $E=60 \mathrm{MPa}, v=0.4, \bar{G}=21.4 \mathrm{MPa}$, $\sigma_{G}=10,7 \mathrm{MPa}$ (i.e. 50 per cent of $\bar{G}$ ) where $E$ is the Young's modulus, $v$ denotes the Poisson's ratio, $\bar{G}$ and $\sigma_{G}$ are the mean value and standard deviation of the shear modulus.

The selected spectrum responses of the platform model, including the case when pin support of the legs simulate a connection of the structure to the subsoil, are shown in Figs. 4 and 5. The spectrum for the non-linear stochastic case is referred to as the mean values of the natural frequencies obtained from a linear stochastic solution.

\section{CONCLUDING REMARKS}

Based on the carried out analysis the following conclusions can be drawn:

1. The structural response is obtained in a frequency-domain by using a linearized form of drag forces, the subsoil stiffness and damping parameters are assumed deterministic 
(a linear stochastic problem) or random variables (a nonlinear stochastic problem).

2. Modal reduction of dynamic equations of motion performed to achieve a reasonable accuracy of structural response includes a few of the lowest modes.

3. The assumption that soil-structure interaction is modelled by pin supports leads to shifting the response spectrum to lower frequency values, compared to spring supports of the platform legs (Fig. 4).

4. The response spectra reveal two main peaks. The first one is related to the characteristic frequency of the wave $(\omega=0.5 \mathrm{rad} / \mathrm{s})$ whereas the second one is linked with the lowest natural frequencies of the structural system $\left(\omega_{01}=1.561 \mathrm{rad} / \mathrm{s}\right.$ for $\beta=0^{\circ}$ and $\omega_{02}=2.162 \mathrm{rad} / \mathrm{s}$ for $\left.\beta=90^{\circ}\right)$.

5. There are series of zero points in the spectra due to those wavelengths which produce the forces on legs that are equal and of opposite sign (Fig. 4 and Fig. 5).

6. The wave load makes a major contribution to dynamic response in comparison to the wind load; the difference does not exceed $10 \%$ (cf. [6]).

7. For the non-linear stochastic case the peak in the response is about twice as great as that in the case of deterministic subsoil parameters. Thus the evaluation of the soilstructure interaction parameters is decisive in the response approximation.

8. In the case of relatively slender members of steel platforms major part of the damping forces of the vibrating system is produced by sea waves. When concrete gravity platforms are considered the subsoil contributes to total damping more significantly (cf. [2], [17], [18]).

9. The analysis presented in the paper refers to more general offshore engineering dynamic systems, thus it may be also applicable to structures supporting offshore wind turbines.

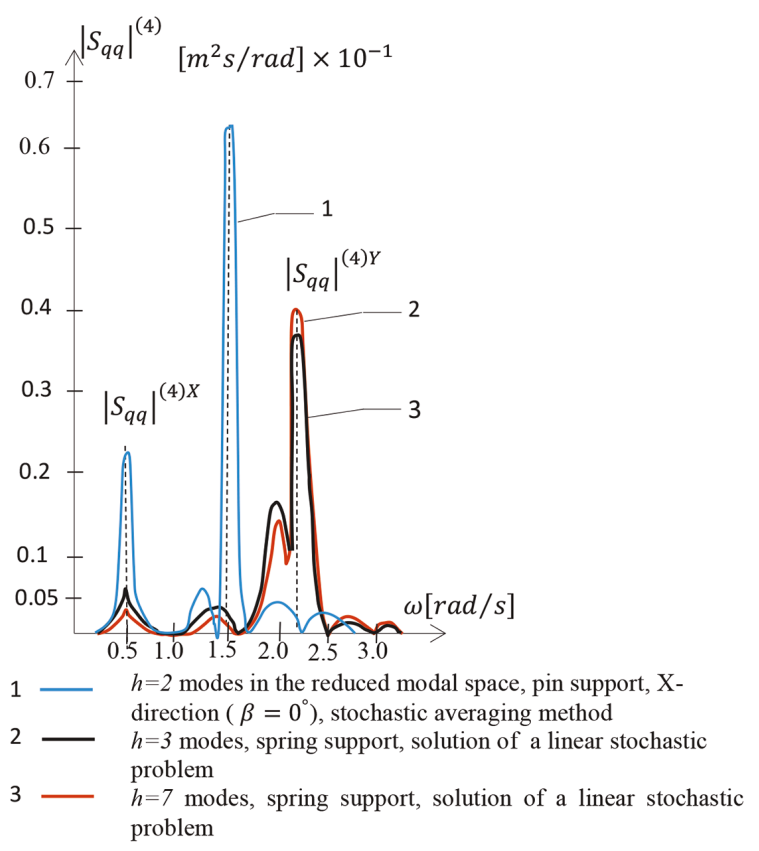

Fig. 4. Spectrum moduli of the displacement response at node (4) due to wave loads

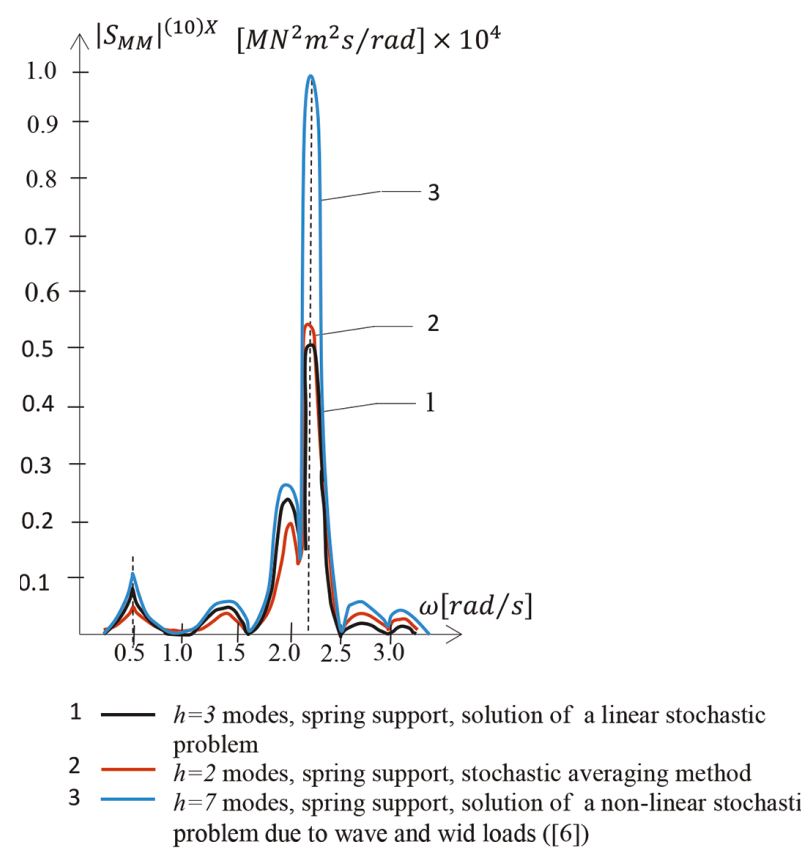

Fig. 5. Spectrum moduli of the response moment at the bottom at node 10 due to wave and wind loads

\section{REFERENCES}

1. Malhotra A.K.,Penzien J.: Response of offshore structures to random wave forces. Journal of the Structural Division, ASCE, 96, Nr ST10, 1970, pp. 2155-2173.

2. Clauss G., Lehmann E., Ostergaard C.: Offshore structures, Vol. ISpringer-Verlag, 1992, Vol. II, 1994.

3. Penzien J., Tseng S.: Three-dimensional dynamic analysis of fixed offshore platforms. Numerical Methods in Offshore Engineering, O.C. Zienkiewicz, R.W. Lewis and K.G. Stagg editors - Chapter 7, J. Wiley \& Sons, New York, 1979

4. Rozmayrnowski B., Mikulski T.: Selected problems of sensitivity and reliability of a jack-up platform. Polish Maritime Research, 1 (97), Vol. 25, 2018, pp. 77-84.

5. DNV-OS-J101: Det Norske Veritas, Design of offshore wind turbine structures. May 2014.

6. Jesien W.: Random Vibrations of the Baltic Drilling Platform Subjected to Wind Loads and Water Waves. Earthquake Engineering and Structural Dynamics, 15, 1987, pp. 595-617.

7. Spanos P.D.: Stochastic linearization in structural dynamics. AMR, 34, No. 1, 1981.

8. Rozmarynowski B.: Averaged Damping in Random Vibrations of the Baltic Drilling Platform. Journal of Sound and Vibration 139(3), 1990, pp. 437-458.

9. Taylor E.R., Rajagopalan A.: Dynamics of offshore structures, Part I: Perturbation analysis. Journal of Sound and Vibration, No. 3, Vol. 83, 1982, pp. 417-431. 
10. RajagopalanA.,Taylor E.R.: Dynamics of offshore structures, Part II: Stochastic averaging analysis. Journal of Sound and Vibration, No 3,Vol. 83, 1982, pp. 401-416.

11. Gierliński J.: RASOS (Reliability Analysis System for Offshore Structures) - Theoretical manual, Version Nov. WS-Atkins, UK, 1991.

12. Rozmarynowski B.: Time-domain response of the Baltic drilling platform under regular sea wave excitation. (in Polish). Archiwum Hydrotechniki, Nr XXXV/3-4, 1988, pp. 251-276

13. Massel St.: Hydrodynamical issues of offshore structures (in Polish), PWN, 1981.

14. Davenport A.G.: The spectrum for horizontal gustiness near the ground in high winds. Quarterly Journal of the Royal Meteorological Society, 87, 1961, pp.194-211..

15. Morison, J.R., O’Brien, M.P., Johnson, J.W. and Schast, S.A.: The forces excerted by surface waves on piles. PetroleumTrans., ASME, 189, 1950, pp. 149-154.

16. Clough R.W. and Penzien J.: Dynamics of Structures. McGrawHill, New York, 1975.

17. Barltrop N.D.P., Adams A.J.: Dynamics of fixed marine structures. Butterworth Heinemann, 1991.

18. Wilson J.F. (Editor): Dynamics of offshore structures. John Wiley\& Sons, 2003.

19. Spanos P.D.: Stochastic analysis of oscillators with non-linear damping. Int. Journal of Non-linear Mechanics, 13, 1978, pp. 249-259.

20. Khas'minskii R.Z.: A limit theorem for the solution of differential equations with random right-hand sides. Theory of Probability and its Applications, 1966, Vol. 11, No. 3, pp. 390-406.

\section{APPENDIX 1}

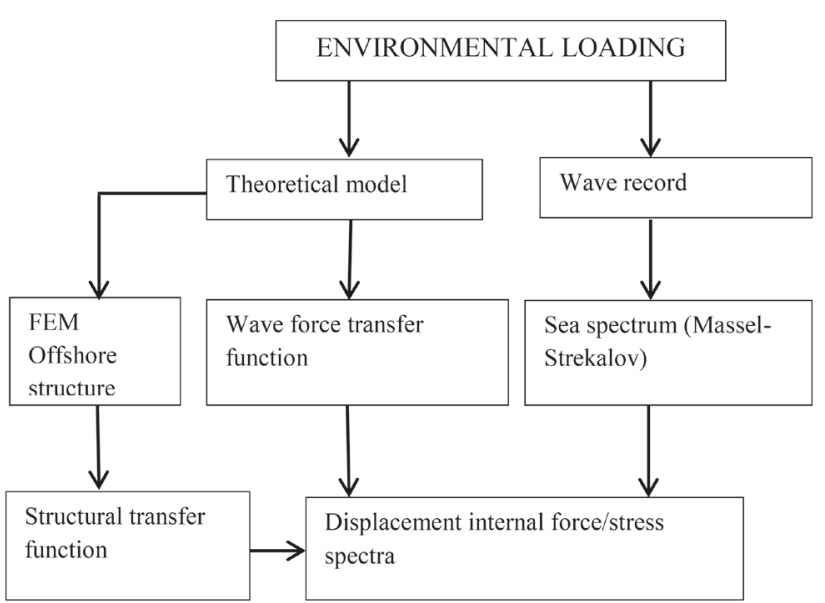

Fig. 6. Calculation flow chart of an internal force and stress spectra

\section{APPENDIX 2}

The Massel-Striekalov spectrum takes the form [13]:

$S_{\eta \eta}(\omega)=\frac{\bar{H}^{2}}{\bar{\omega}}\left\{0.38 \exp \left[-35\left(\frac{\omega}{\bar{\omega}}-0.8\right)^{2}\right]+0.22\left(\frac{\omega}{\bar{\omega}}\right)^{-5} \exp \left[-1.34\left(\frac{\omega}{\bar{\omega}_{P}}\right)^{-8}\right]\right\}$

(A1)

with parameters $\bar{H}, \bar{\omega}$ indicating the mean wave height and frequency of the wave field.

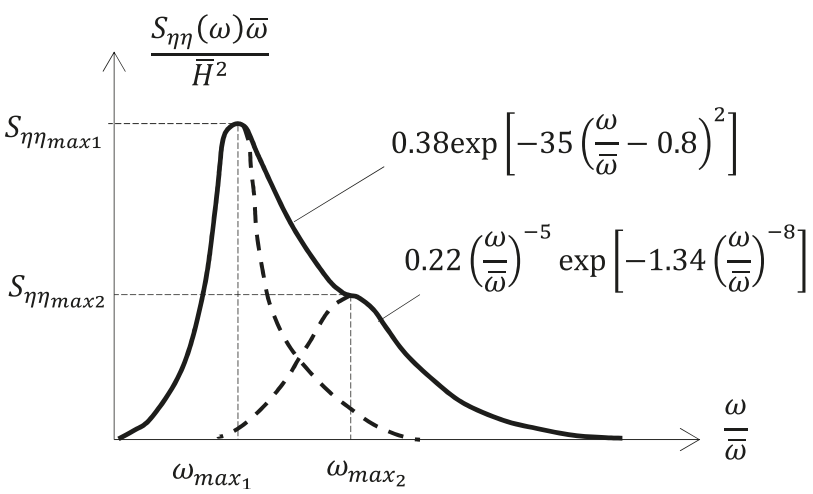

Fig. 7. One-dimensional wave spectrum defined by Striekalov and Massel [13]

\section{APPENDIX 3}

A spectral density function of the wind velocity fluctuations considered a one-dimensional stationary Gaussian process with zero mean value is given by Davenport [14]:

$$
S_{v^{\prime} v^{\prime}}(\omega)=\frac{2 K L^{2} \omega}{\pi\left[1+\left(\frac{\omega L}{2 \pi \bar{v}_{10}}\right)^{2}\right]^{4 / 3}}
$$

where $K$ is the surface drag coefficient for open sea areas, $L$ is the length scale of turbulence ( $L=1200 \mathrm{~m}$ according to Davenport), $\bar{v}_{10}$ represents the mean speed at a height of $10 \mathrm{~m}$ above the surface, $\omega$ is the frequency. The graph of Eq. (A2) is presented in Fig. 8.

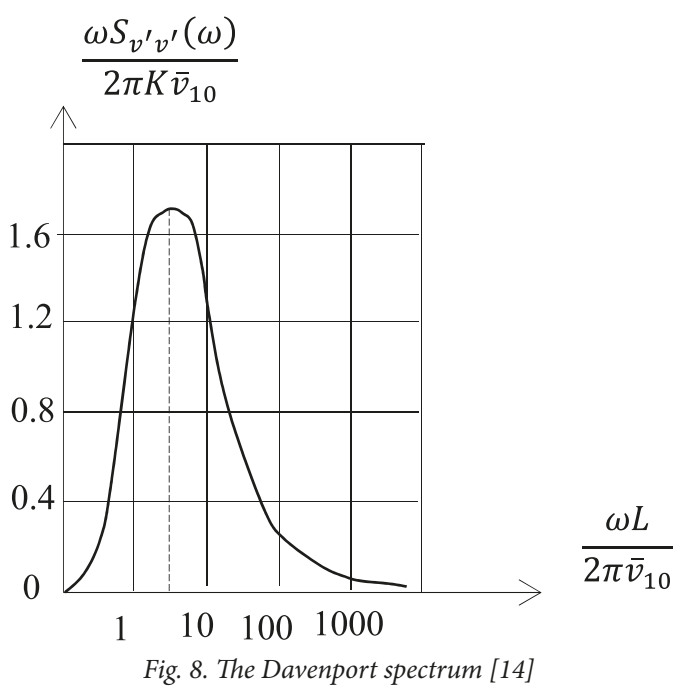




\section{CONTACT WITH THE AUTHOR}

Bogdan Rozmarynowski

e-mail: bogrozma@pg.edu.pl

Gdańsk University of Technology

11/12 Narutowicza St.

80-233 Gdańsk

Poland 